\title{
Antithymocyte globulin therapy for patients with recent-onset type 1 diabetes: 2 year results of a randomised trial
}

\author{
Stephen E. Gitelman ${ }^{1,2}$ • Peter A. Gottlieb ${ }^{3}$ - Eric I. Felner ${ }^{4}$ - Steven M. Willi ${ }^{5,6}$ • \\ Lynda K. Fisher $^{7}$ • Antoinette Moran ${ }^{8}$ - Michael Gottschalk ${ }^{9}$ - Wayne V. Moore ${ }^{10}$. \\ Ashley Pinckney ${ }^{11}$ - Lynette Keyes-Elstein ${ }^{11}$ - Kristina M. Harris ${ }^{12}$ • Sai Kanaparthi ${ }^{12}$.

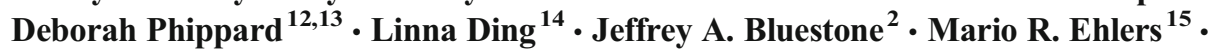 \\ the ITN START Study Team
}

Received: 8 November 2015 / Accepted: 17 February 2016/Published online: 6 April 2016

(C) Springer-Verlag Berlin Heidelberg 2016

\begin{abstract}
Aims/hypothesis Type 1 diabetes results from T cell mediated destruction of beta cells. We conducted a trial of antithymocyte globulin (ATG) in new-onset type 1 diabetes (the Study of Thymoglobulin to ARrest T1D [START] trial). Our goal was to evaluate the longer-term safety and efficacy of ATG in preserving islet function at 2 years.

Methods A multicentre, randomised, double-blind, placebocontrolled trial of $6.5 \mathrm{mg} / \mathrm{kg}$ ATG (Thymoglobulin) vs placebo in patients with new-onset type 1 diabetes was conducted at seven university medical centres and one Children's Hospital in
\end{abstract}

A full list of the ITN Start Study Team is available in the electronic supplementary material (ESM).

Electronic supplementary material The online version of this article (doi:10.1007/s00125-016-3917-4) contains peer-reviewed but unedited supplementary material, which is available to authorised users.

Stephen E. Gitelman

sgitelma@ucsf.edu

1 Division of Pediatric Endocrinology, University of California San Francisco, Mission Hall, 550 16th Street, 4th Floor, Box 0434, San Francisco, CA 94158-2549, USA

2 Diabetes Center, University of California San Francisco, San Francisco, CA, USA

3 Barbara Davis Center, University of Colorado, Aurora, CO, USA

4 Division of Pediatric Endocrinology, Emory University School of Medicine, Atlanta, GA, USA

5 Division of Endocrinology and Diabetes, Children's Hospital of Philadelphia, Philadelphia, PA, USA

6 Department of Pediatrics, Perelman School of Medicine at the University of Pennsylvania, Philadelphia, PA, USA

the USA. The site-stratified randomisation scheme was computer generated at the data coordinating centre using permuted-blocks of size 3 or 6 . Eligible participants were between the ages of 12 and 35, and enrolled within 100 days from diagnosis. Subjects were randomised to $6.5 \mathrm{mg} / \mathrm{kg}$ ATG (thymoglobulin) vs placebo in a 2:1 ratio. Participants were blinded, and the study design included two sequential patient-care teams: an unblinded studydrug administration team (for the first 8 weeks), and a blinded diabetes management team (for the remainder of the study). Endpoints assessed at 24 months included meal-stimulated C-peptide AUC, safety and immunological responses.

7 of Los Angeles, Los Angeles, CA, USA

8 Department of Pediatrics, Division of Pediatrics Endocrinology, University of Minnesota, Minneapolis, MN, USA

9 Department of Pediatrics, University of California San Diego, San Diego, CA, USA

10 Department of Pediatrics, Division of Pediatric Endocrinology, Children's Mercy Hospital and University of Missouri-Kansas City, Kansas City, MO, USA

11 Rho Federal Systems Division, Chapel Hill, NC, USA

12 Immune Tolerance Network, Bethesda, MD, USA

13 Present address: Precision for Medicine, Bethesda, MD, USA

14 National Institute of Allergy and Infectious Diseases, Bethesda, MD, USA

15 Immune Tolerance Network, San Francisco, CA, USA 
Results Fifty-eight patients were enrolled; at 2 years, 35 assigned to ATG and 16 to placebo completed the study. The pre-specified endpoints were not met. In post hoc analyses, older patients (age 22-35 years) in the ATG group had significantly greater C-peptide AUCs at 24 months than placebo patients. Using complete preservation of baseline C-peptide at 24 months as threshold, nine of 35 ATG-treated participants (vs $2 / 16$ placebo participants) were classified as responders; nine of 11 responders (67\%) were older. All participants reported at least one adverse event (AE), with 1,148 events in the 38 ATG participants vs 415 in the 20 placebo participants; a comparable number of infections were noted in the ATG and placebo groups, with no opportunistic infections nor difficulty clearing infections in either group. Circulating $\mathrm{T}$ cell subsets depleted by ATG partially reconstituted, but regulatory, naive and central memory subsets remained significantly depleted at 24 months. Beta cell autoantibodies did not change over the 24 months in the ATG-treated or placebo participants. At 12 months, ATG-treated participants had similar humoral immune responses to tetanus and HepA vaccines as placebo-treated participants, and no increased infections. Conclusions/interpretation A brief course of ATG substantially depleted $\mathrm{T}$ cell subsets, including regulatory cells, but did not preserve islet function 24 months later in the majority of patients with new-onset type 1 diabetes. ATG preserved C-peptide secretion in older participants, which may warrant further study.

Trial registration: ClinicalTrials.gov NCT00515099

Public data repository: START datasets are available in TrialShare www.itntrialshare.org

Funding: National Institute of Allergy and Infectious Diseases (NIAID) of the National Institutes of Health (NIH). The trial was conducted by the Immune Tolerance Network (ITN).

Keywords ATG · Beta cells · C-peptide · START trial · T cells $\cdot$ Thymoglobulin $\cdot$ Tregs $\cdot$ Type 1 diabetes

$\begin{array}{ll}\text { Abbreviations } \\ \text { AE } & \text { Adverse event } \\ \text { ATG } & \text { Antithymocyte globulin } \\ \text { CRS } & \text { Cytokine release syndrome } \\ \text { EBV } & \text { Epstein Barr virus } \\ \text { G-CSF } & \text { Granulocyte colony-stimulating factor } \\ \text { IA-2 } & \text { Protein tyrosine phosphatase-like protein } \\ \text { ICA } & \text { Islet-cell antigens } \\ \text { ITT } & \text { Intent to treat } \\ \text { MMTT } & \text { Mixed meal tolerance test } \\ \text { SAE } & \text { Serious adverse event } \\ \text { START } & \text { Study of Thymoglobulin to ARrest T1D } \\ \text { Tcm } & \text { Central memory T cell } \\ \text { Tem } & \text { Effector memory T cell }\end{array}$
Tn
Naive T cell
Treg Regulatory T cell

\section{Introduction}

Type 1 diabetes results primarily from $T$ cell mediated destruction of insulin-producing beta cells. At diagnosis, significant beta cell mass remains and, if preserved, can improve glycaemic control and reduce risk for long-term complications. Therapies directed against $\mathrm{T}$ cells can alter the course of disease, but to date have had only initial limited success in beta cell preservation [1-9].

We previously reported the 1-year results of a trial investigating a 4-day course of antithymocyte globulin (ATG) administered to patients with new-onset type 1 diabetes (the Study of Thymoglobulin to ARrest T1D [START] trial) [10]. We found that treatment with ATG resulted in profound depletion of most subsets of $\mathrm{CD} 4^{+}$and $\mathrm{CD} 8^{+} \mathrm{T}$ cells, but there was no significant depletion of effector memory $\mathrm{T}$ cells, which are considered the principal class of pathogenic effector T cells in this disease. We also observed a high incidence of moderate to severe cytokine release syndrome (CRS) and serum sickness in ATG-treated participants. The trial did not reach the primary outcome (preservation of meal-stimulated C-peptide at 12 months in the intent-to-treat [ITT] population), but there was a suggestion of stabilisation in beta cell loss after 6 months, as well as possible benefit in adults compared with children. The START patients were followed for an additional 12 months out to 24 months, and we now report the efficacy, safety and immunological response to ATG at 2 years in this study.

\section{Methods}

Trial Design The START trial study design and results of the primary outcome at 1 year have been reported previously [10], and the study protocol is available at www.itntrialshare.org (accessed 25 February 2016). Briefly, screening, enrolment and subsequent study visits occurred at 11 sites in the USA, with a total of 58 participants enrolled in a randomised, placebo-controlled, blinded, phase 2 clinical trial. The primary outcome was at 12 months, with pre-specified secondary endpoint at 24 months reported herein. The trial was registered with ClinicalTrials.gov (NCT00515099). Investigational review boards approved the study protocol at each study site.

Participants and eligibility criteria Eligible participants were $12-35$ years of age at time of screening; $<100$ days from diagnosis at the time of enrolment; positive for at least one diabetes-associated autoantibody (insulin, GAD, protein 
tyrosine phosphatase-like protein [IA-2], or islet-cell antigens [ICA]); had peak stimulated C-peptide of $>0.4 \mathrm{nmol} / 1$ during a mixed meal tolerance test (MMTT); and had prior serological evidence of Epstein Barr virus (EBV) infection. Exclusion criteria included evidence of tuberculosis, hepatitis B or C, human immunodeficiency virus, or any active infection; significant cardiac disease; leucopenia or thrombocytopenia; prior treatment with rabbit ATG, or known hypersensitivity to rabbit proteins; liver or renal dysfunction; ongoing use of diabetes medications other than insulin; recent inoculation with a live vaccine; and lactating or pregnant women. All participants gave informed consent.

Randomisation and masking Eligible participants were randomly assigned $2: 1$ to ATG $(n=38)$ or placebo $(n=20)$. The site-stratified randomisation scheme was computer generated at the data coordinating centre using permuted-blocks of size 3 or 6 . All participants were masked and initially managed by an unmasked drug management team, which managed all aspects of the study until month 3. Thereafter, to maintain masking for diabetes management through the remainder of the study, the participants received diabetes management from an independent masked study physician and nurse educator. While participants were given the overall study results after year 1, participants and staff remained masked to the individual study assignment and data until all participants completed the month 24 visit and data lock had occurred. Data reported herein were obtained prior to unmasking study participants or staff to treatment assignment.

Interventions All participants were admitted to a clinical research centre for continuous observation during the infusion period and discharged $24 \mathrm{~h}$ after completion of the last infusion. The ATG group received a total dose of $6.5 \mathrm{mg} / \mathrm{kg}$ ATG (Thymoglobulin, Genzyme, Cambridge, MA, USA), with $0.5 \mathrm{mg} / \mathrm{kg}$ on day 1 , and $2 \mathrm{mg} / \mathrm{kg}$ on days $2-4$; placebo participants received saline $(154 \mathrm{mmol} / \mathrm{l} \mathrm{NaCl})$. All participants were pre-medicated with diphenhydramine and acetaminophen; participants in the ATG group also received i.v. methylprednisolone $0.5 \mathrm{mg} / \mathrm{kg}$ prior to the infusion, and $0.25 \mathrm{mg} / \mathrm{kg}$ $12 \mathrm{~h}$ later on days $1-3$ (day $4: 0.25 \mathrm{mg} / \mathrm{kg}$ before infusion, later dose optional); the placebo group received matching saline infusions. Serum sickness was managed with prednisone with a maximum dose of $1.5 \mathrm{mg} \mathrm{kg}^{-1}$ day $^{-1}$ on days $1-3$, with rapid tapering thereafter. The ATG group received prophylaxis with trimethoprim-sulfamethoxazole, and acyclovir if previous herpes or varicella exposure, for at least 3 months and until CD 4 counts were $>200$ cells $/ \mu$ l; the placebo group received placebo tablets for 3 months. All participants received intensive diabetes management with the goal to achieve ADA $\mathrm{HbA}_{1 \mathrm{c}}$ and glycaemic targets for age. Four-h MMTTs were repeated at 6-month intervals out to 24 months.
Laboratory tests Biochemical autoantibodies were assayed at the Barbara Davis Center (Aurora, CO, USA) using radioimmunobinding assays, and ICA was measured at the University of Florida (FL, USA). C-peptide and $\mathrm{HbA}_{1 \mathrm{c}}$ were measured at the Northwest Lipid Research Laboratory (Seattle, WA, USA) as described previously [10]. Immunophenotyping of lymphocyte and antigen presenting cell subsets was performed in real-time using multi-colour flow cytometry (antibody panel configuration in the electronic supplementary material [ESM] Table 1) using a Cytomics FC500 flow cytometer (Beckman Coulter, Brea, CA, USA); manual sequential gating was done in Flowjo (TreeStar, Ashland, OR, USA).

Statistical analysis Details of the statistical plan, including the primary endpoint analysis at 12 months, are described in our prior report [10]. The primary endpoint analysis was a comparison of the change in the mean $2 \mathrm{~h}$ C-peptide AUC from baseline, adjusted for the baseline C-peptide response, in the ATG vs placebo groups 12 months after study enrolment. Pre-specified secondary outcomes at 24 months included the $2 \mathrm{~h}$ and $4 \mathrm{~h} \mathrm{C}$-peptide AUC; changes of C-peptide AUC over time; insulin use; proportion of participants who are exogenous insulin-free; major hypoglycaemic events; $\mathrm{HbA}_{1 \mathrm{c}}$ levels; and frequency and severity of adverse events (AEs) in the ATG vs placebo groups. Sample size calculations were described in our prior report [10].

All randomised participants who received any dose of study treatment $(n=58)$ were used in the ITT analysis for the secondary endpoints. Seven participants in the ITT population did not have an MMTT at month 24 (three ATG, four placebo). Missing month-24 MMTTs were imputed as described in the ESM methods. For analysis, C-peptide AUC values were transformed to $\log _{e}(\mathrm{AUC}+1)$ values. An F-test derived from an ANCOVA with baseline $\log _{e}(\mathrm{AUC}+1)$ value as a covariate was used to compare treatment groups at month 24. Means and summary statistics are presented on the untransformed scale. Adjusted means were based on models fit to untransformed AUC values. The $p$ values were derived from models using $\log _{e}(\mathrm{AUC}+1)$ as the outcome and using the KenwardRogers approximation for inference. Methods of computation for 2 and $4 \mathrm{~h} \mathrm{C}$-peptide AUC can be found in the ESM methods.

Secondary inferential analyses on $\mathrm{HbA}_{1 \mathrm{c}}$ and insulin use were based on ANCOVA models at each time point with adjustment for baseline levels. Fisher's exact test was used to compare the number of participants who were insulin independent and who had a hypoglycaemic event out to month 24 . For any secondary and exploratory analyses, corrections were not made for multiple comparisons. SAS version 9.3 (Carey, $\mathrm{NC}$, USA) was used for data analysis.

Flow cytometry data were analysed using absolute counts (cells $/ \mu \mathrm{l})$. One-sided paired $t$ test was applied to test whether 
the absolute cell counts at month 24 were significantly lower than the absolute counts at baseline within the ATG group. This analysis was exploratory and no adjustments were made for multiple comparisons.

\section{Results}

Participants The participant experience in the trial is shown in ESM Fig. 1; 38 participants were randomised to the treatment group and 20 to the placebo group. Of these, 35 in the treatment group and 16 in the placebo group completed the 2-year assessment. Demographic and baseline characteristics were comparable between the ATG and placebo groups [10]. When the baseline characteristics were subdivided by age group (younger group, 12-21 years; older group, 22-35 years), the ATG and placebo arms within each age group were generally balanced (ESM Table 2). The younger group had slightly higher baseline $2 \mathrm{~h}$ AUC and peak C-peptide values and higher baseline insulin use (ESM Table 2).

Change in C-peptide secretion There was no significant difference in the primary outcome, the change from baseline to 12 months in the $2 \mathrm{~h}$ C-peptide AUC in response to an MMTT, in the ATG vs placebo groups [10]. Both the ATG and placebo groups showed further decline over the second 12-month period (Fig. 1a), with the ATG group exhibiting a mean change from baseline at 24 months of $-0.27 \mathrm{nmol} / 1$ (95\% CI -0.373 , $-0.171)$ vs a mean decrease in the placebo group of $-0.32 \mathrm{nmol} / \mathrm{l}(95 \% \mathrm{CI}-0.473,-0.174 ; p=0.38)$. Notably, C-peptide levels did not stabilise in the ATG group as a whole beyond 12 months (Fig. 1a). However, the rate of C-peptide decline was slower in the older ATG-treated participants (defined as ages 22-35 years) compared with the younger participants (12-21 years), an effect that persisted out to 24 months (Fig. 1b, c). After adjustment for baseline, the mean change in $2 \mathrm{~h}$ C-peptide AUC from baseline to 24 months in the older ATG participants was $-0.075 \mathrm{nmol} / 1(95 \% \mathrm{CI}-0.286,0.136) \mathrm{vs}$ $-0.401(95 \% \mathrm{CI}-0.684,-0.135)$ in the older placebo participants $(p=0.026)$. In the younger ATG participants, the mean change was $-0.276 \mathrm{nmol} / 1(95 \% \mathrm{CI}-0.423,-0.130)$ vs $-0.299(95 \% \mathrm{CI}-0.522,-0.0762)$ in the younger placebo participants $(p=0.84)$.

In light of the apparent treatment benefit in the older age group, we considered the possibility that there may have been a subset of 'responders' with preservation of C-peptide secretion at 24 months. To evaluate this, we performed a series of post hoc analyses with different responder thresholds to identify a threshold that included a subset of ATG-treated participants but no or few placebo-treated participants. In a plot of age vs percentage change from baseline in the $2 \mathrm{~h}$ C-peptide AUC, a cluster of nine participants (24\%) in the ATG arm showed no loss of baseline C-peptide at 24 months $(>0 \%$
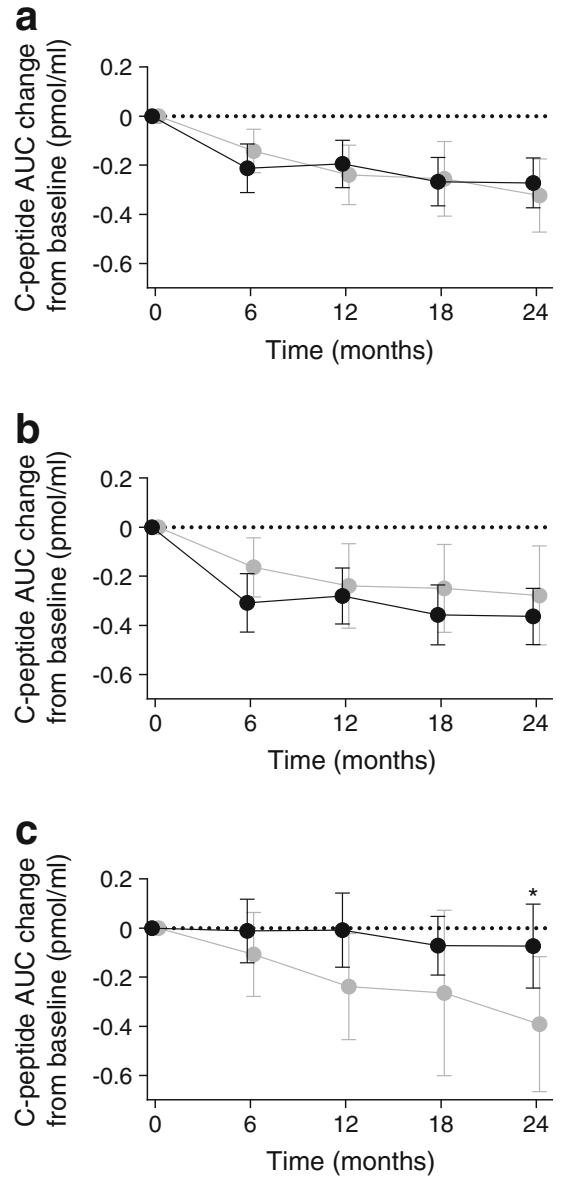

Fig. 1 Change in C-peptide AUC from baseline to 24 months in participants assigned to ATG and placebo in the ITT sample. (a) Change in $2 \mathrm{~h} \mathrm{C}$-peptide AUC in all participants. The number of evaluable participants $(n)$ at each time point is shown in ESM Fig. 1. (b) Change in $2 \mathrm{~h} \mathrm{C}$-peptide AUC in participants aged 12-21 years. (ATG, $n=26$; placebo, $n=12$ ). (c) Change in $2 \mathrm{~h} \mathrm{C}$-peptide AUC in participants aged 22-35 years (ATG, $n=12$; placebo, $n=8$ ). Black, ATG; grey, placebo. Data were analysed by fitting ANCOVA models with adjustment for baseline levels and plotted as unadjusted means $\pm 95 \% \mathrm{CI} ; p$ values are two-sided; $* p<0.05$

change) and were designated responders; two participants $(10 \%)$ in the placebo group also met this criterion (Fig. 2a, b). In the ATG arm, 6 (67\%) of the responders were in the older cohort while none of the responders in the placebo arm were older participants ( $p=0.044)$. Within the ATG group, six of 12 participants in the older cohort were responders compared with three of 23 participants in the younger cohort $(p=0.038)$, consistent with the finding that the older ATG cohort appeared to have a treatment benefit both compared to older placebo participants and to younger participants within the ATG group.

Glycaemic control was well maintained in both treatment groups, but $\mathrm{HbA}_{1 \mathrm{c}}$ did show a steady rise from month 12 to 24 , with no significant difference between the ATG and placebo groups at month 24 ( $p=0.157$; ESM Fig. $2 \mathrm{a}$ ). There was no difference in exogenous insulin use between the ATG and 

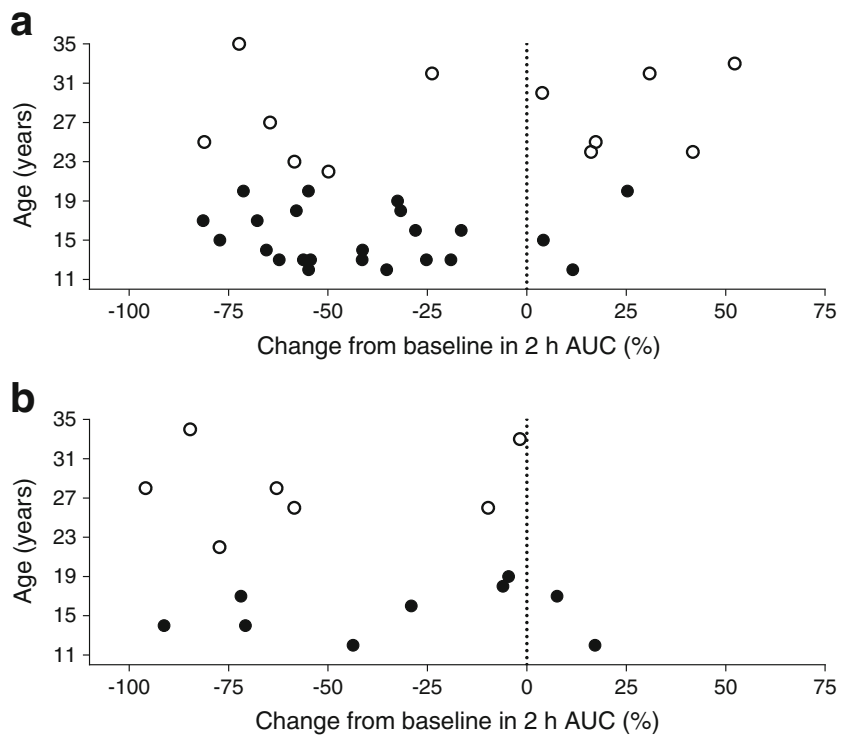

Fig. 2 Responder analysis based on preservation of baseline C-peptide secretion at 2 years. The $\%$ change in $2 \mathrm{~h} \mathrm{C}$-peptide AUC from baseline to 2 years was plotted for each participant as a function of age (black circles, younger participants [12-21 years]; white circles, older participants [2235 years]); (a) ATG arm; (b) placebo arm. Participants to the right of the dotted line $(>0 \%$ change $)$ are denoted 'responders'

placebo groups ( $p=0.136$; ESM Fig. $2 \mathrm{c}$ ); one participant (ATG) was insulin-free at 24 months. The number of participants who had at least one hypoglycaemic event (defined as a glucose concentration $<3.06 \mathrm{mmol} / \mathrm{l}$, or symptomatic) out to month 24 was not significantly different between the two groups (ATG 32/38, placebo 16/20; Table $1 ; p=0.764$ ). One participant (placebo) experienced diabetic ketoacidosis during the 24-month study period. We also evaluated the difference in metabolic outcomes between the older and younger participants in the ATG-treated and placebo groups (ESM Fig. 2b, d). The older ATG-treated cohort had significantly lower $\mathrm{HbA}_{1 \mathrm{c}}$ than the younger ATG-treated group $(p=0.0042)$, and had lower exogenous insulin needs $(p=0.0081)$. The overall higher exogenous insulin needs and higher $\mathrm{HbA}_{1 \mathrm{c}}$ in the younger cohort compared with the older cohort are not unexpected, based on prior studies [11,12]. We did not find a significant difference in major hypoglycaemia events between the older and younger ATG-treated participants.

Safety All participants reported at least one AE, with 1,148 events in the 38 ATG participants vs 415 in the 20 placebo participants (Table 1). Of these events, 387 AEs in 35 participants in the ATG group and 164 AEs in 17 placebo participants occurred in the second year (ESM Table 3). During the second year of the study, there were a total of seven serious adverse events (SAEs) in five participants in the ATG group and three SAEs in two participants in the placebo group (ESM Table 4). Almost all participants in the treatment group experienced CRS during the ATG infusion, and all experienced serum sickness 1-2 weeks later, with complete resolution of all symptoms within the first 3-4 weeks after therapy. A comparable number of infections per participant was noted in the ATG and placebo groups, with median time to infection of 291 days in the ATG group and 287 days in the placebo group. There were no opportunistic infections or difficulty clearing infections in either group. No EBV or cytomegalovirus reactivation was noted clinically or via PCR assessment. AEs were also evaluated by age group of the participants, grouping them into younger (12-21 years) and older (22-35 years) cohorts, and we did not find an appreciable difference in the nature or severity of the early events (CRS and serum sickness) or later findings between the younger and older ATG-treated participants (ESM Table 5).

Mechanistic results Mean absolute counts of total $\mathrm{CD} 4^{+}$and $\mathrm{CD}^{+} \mathrm{T}$ cells fell precipitously in peripheral blood after ATG treatment, and slowly reconstituted over the ensuing months (Fig. 3). Although in the normal range, $\mathrm{CD}^{+} \mathrm{T}$ cells remained significantly below their baseline levels at 24 months $(p<0.001)$; $\mathrm{CD}^{+}$cells recovered faster and more fully, but also remained significantly below their baseline levels at 24 months $\left(p=0.003\right.$ ) (Fig. 3a, b). $\mathrm{CD}^{+}$and $\mathrm{CD} 8^{+}$naive $(\mathrm{Tn})$ and central memory $(\mathrm{Tcm}) \mathrm{T}$ cells, and $\mathrm{CD} 4^{+}$regulatory T cells (Tregs) were substantially depleted early and, although partially reconstituted, absolute counts in the peripheral blood remained significantly lower at 24 months compared to baseline $(p<0.001)$ (Fig. 3 c, d). In contrast, $\mathrm{CD}^{+}$and $\mathrm{CD} 8^{+}$ effector memory (Tem) $\mathrm{T}$ cells were not significantly depleted by ATG (data not shown). These immunological changes were not noted in the placebo group (data not shown), indicating this is a treatment effect, and not simply due to the natural progression of the disease.

We showed previously that depletion of Tregs and preservation of Tem cells by ATG resulted in an unfavourable Treg/Tem ratio [10]. These $T$ cell changes persisted into the second year despite partial reconstitution and may account for the continued inability of ATG treatment to preserve C-peptide at 24 months. Mechanistic analyses of the older and younger cohorts did not reveal significant differences in these specific lymphocyte subsets that could account for the age-related difference in beta cell preservation. There were also no significant changes in these immune cell subsets between responders and non-responders as defined above (not shown).

Autoantibodies and vaccine responses Over the 24-month study period, there were no significant changes from baseline in mean titres of $\operatorname{IgG}$ that recognise the autoantigens GAD, IA-2, insulin, ICA, and zinc transporter 8 within either treatment arm, and there were no significant between-group differences at any time point (data not shown).

To determine the impact of ATG on immune function, T celldependent humoral responses to a neoantigen (hepatitis $\mathrm{A}$ ) and 
Table 1 AEs in $15 \%$ or more of participants up to month 24

\begin{tabular}{|c|c|c|c|c|}
\hline & ATG participants $(n=38)$ & Events & Placebo participants $(n=20)$ & Events \\
\hline Total number of events & $38(100 \%)$ & 1148 & $20(100 \%)$ & 415 \\
\hline Grade 1 & $38(100 \%)$ & 350 & $18(90 \%)$ & 179 \\
\hline Grade 2 & $37(97 \%)$ & 604 & $18(90 \%)$ & 200 \\
\hline Grade 3 & $31(82 \%)$ & 112 & $12(60 \%)$ & 30 \\
\hline Grade 4 & $38(100 \%)$ & 78 & $4(20 \%)$ & 5 \\
\hline Grade 5 & 0 & 0 & 0 & 0 \\
\hline Metabolism and nutrition disorders & $35(92 \%)$ & 513 & $16(80 \%)$ & 206 \\
\hline Hypoglycaemia & $32(84 \%)$ & 495 & $16(80 \%)$ & 201 \\
\hline Hyperglycaemia & $8(21 \%)$ & 12 & $1(5 \%)$ & 4 \\
\hline Infections and infestations & $29(76 \%)$ & 88 & $14(70 \%)$ & 33 \\
\hline Upper respiratory tract infection & $16(42 \%)$ & 32 & $7(35 \%)$ & 16 \\
\hline Viral infection & $5(13 \%)$ & 11 & $3(15 \%)$ & 3 \\
\hline Blood and lymphatic system disorders & $38(100 \%)$ & 123 & $4(20 \%)$ & 6 \\
\hline CD4 lymphocytes decreased & $38(100 \%)$ & 38 & 0 & 0 \\
\hline Lymphopenia & $38(100 \%)$ & 48 & 0 & 0 \\
\hline Leucopenia & $14(37 \%)$ & 20 & 0 & 0 \\
\hline Neutropenia & $9(24 \%)$ & 10 & $2(10 \%)$ & 4 \\
\hline Immune system disorders & $38(100)$ & 84 & $3(15 \%)$ & 3 \\
\hline CRS & $37(97 \%)$ & 37 & $1(5 \%)$ & 1 \\
\hline Serum sickness & $38(100 \%)$ & 39 & 0 & 0 \\
\hline Seasonal allergy & $7(18 \%)$ & 8 & $2(10 \%)$ & 2 \\
\hline Skin and subcutaneous tissue disorders & $26(68 \%)$ & 44 & $10(50 \%)$ & 26 \\
\hline Rash & $7(18 \%)$ & 8 & $2(10 \%)$ & 3 \\
\hline Acne & $7(18 \%)$ & 8 & $1(5 \%)$ & 1 \\
\hline Pruritus & $4(11 \%)$ & 4 & $3(15 \%)$ & 4 \\
\hline Dermatitis contact & $2(5 \%)$ & 2 & $3(15 \%)$ & 3 \\
\hline Gastrointestinal disorders & $24(63 \%)$ & 51 & $11(55 \%)$ & 20 \\
\hline Nausea & $7(18 \%)$ & 8 & $5(25 \%)$ & 7 \\
\hline Diarrhoea & $5(13 \%)$ & 5 & $3(15 \%)$ & 3 \\
\hline Vomiting & $3(8 \%)$ & 4 & $3(15 \%)$ & 3 \\
\hline Dyspepsia & $2(5 \%)$ & 2 & $3(15 \%)$ & 3 \\
\hline Nervous system disorders & $24(63 \%)$ & 41 & $11(55 \%)$ & 23 \\
\hline Headache & $18(47 \%)$ & 25 & $10(50 \%)$ & 15 \\
\hline General disorders and administration site conditions & $26(68 \%)$ & 36 & $8(40 \%)$ & 18 \\
\hline Fatigue & $3(8 \%)$ & 4 & $4(20 \%)$ & 5 \\
\hline Pyrexia & $6(16 \%)$ & 6 & $1(5 \%)$ & 1 \\
\hline Influenza like illness & $2(5 \%)$ & 2 & $3(15 \%)$ & 3 \\
\hline Respiratory, thoracic and mediastinal disorders & $23(61 \%)$ & 42 & $11(55 \%)$ & 26 \\
\hline Oropharyngeal pain & $9(24 \%)$ & 13 & $5(25 \%)$ & 6 \\
\hline Cough & $8(21 \%)$ & 10 & $4(20 \%)$ & 5 \\
\hline Nasal congestion & $3(8 \%)$ & 4 & $3(15 \%)$ & 4 \\
\hline Rhinorrhoea & $1(3 \%)$ & 1 & $3(15 \%)$ & 3 \\
\hline Musculoskeletal and connective tissue disorders & $14(37 \%)$ & 26 & $11(55 \%)$ & 20 \\
\hline Back pain & $4(11 \%)$ & 5 & $5(25 \%)$ & 6 \\
\hline Arthralgia & $4(11 \%)$ & 4 & $3(15 \%)$ & 4 \\
\hline Musculoskeletal pain & $1(3 \%)$ & 1 & $3(15 \%)$ & 3 \\
\hline Psychiatric disorders & $9(24 \%)$ & 15 & $7(35 \%)$ & 12 \\
\hline Depression & $4(11 \%)$ & 7 & $4(20 \%)$ & 4 \\
\hline Vascular disorders & $4(11 \%)$ & 6 & $5(25 \%)$ & 5 \\
\hline Hypotension & 0 & 0 & $3(15 \%)$ & 3 \\
\hline
\end{tabular}



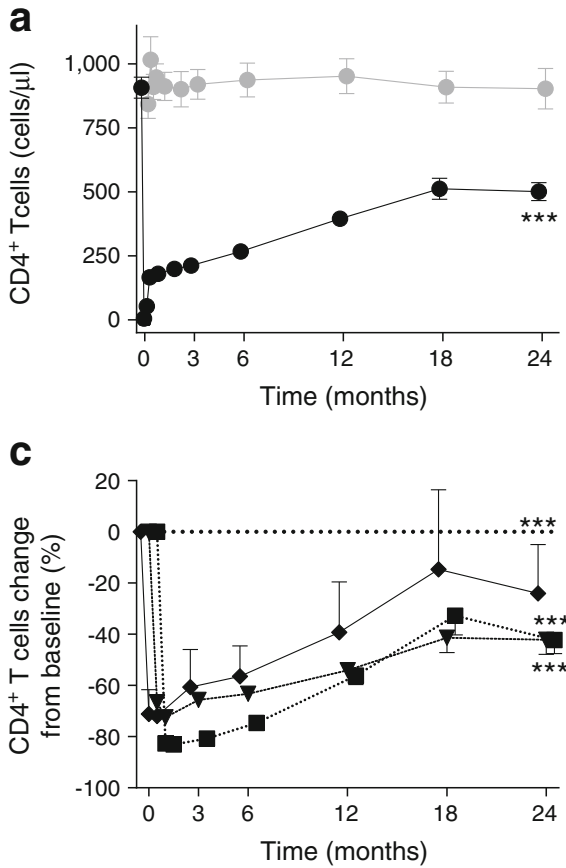

Fig. 3 Changes in $\mathrm{T}$ cell subsets from baseline to 24 months. (a) Absolute counts of total $\mathrm{CD}^{+}{ }^{+} \mathrm{T}$ cells. (b) Absolute counts of total $\mathrm{CD}^{+} \mathrm{T}$ cells. (c) Percentage change from baseline of naive $\mathrm{CD} 4^{+} \mathrm{T}$ cells ( Tn; $\mathrm{CD}^{+} \mathrm{CD}^{2} 5 \mathrm{RA}^{+} \mathrm{CD} 45 \mathrm{RO}^{-} \mathrm{CD}^{2} 2 \mathrm{~L}^{\mathrm{hi}}$ ); central memory $\mathrm{CD} 4^{+} \mathrm{T}$ cells (Tcm; $\mathrm{CD}^{+}{ }^{+} \mathrm{CD} 45 \mathrm{RA}^{-} \mathrm{CD} 45 \mathrm{RO}^{+} \mathrm{CD}^{-} \mathrm{L}^{\mathrm{hi}}$ ); and regulatory $\mathrm{CD} 4^{+} \mathrm{T}$ cells (Treg; $\mathrm{CD} 4{ }^{+} \mathrm{CD} 127^{-/ \mathrm{lo}} \mathrm{CD} 25^{\mathrm{hi}}$ ). (d) Percentage change from baseline of $\mathrm{CD}^{+}$Tn cells $\left(\mathrm{CD}^{+}{ }^{+} \mathrm{CD} 45 \mathrm{RA}^{+} \mathrm{CD} 45 \mathrm{RO}^{-} \mathrm{CD} 62 \mathrm{~L}^{\mathrm{hi}}\right)$ and $\mathrm{CD} 8^{+} \mathrm{Tcm}$

recall antigen (tetanus toxoid) were assessed at baseline, after treatment and prior to vaccination at month 12 , and 3 months after vaccination at month 15 in the ATG and placebo groups. Study participants who tested positive for reactivity to hepatitis A before vaccination at month 12 or for whom samples were not available were excluded from the analysis (22 participants in the ATG arm and 11 participants in the placebo arm). There were no significant differences in the proportion of responders between the ATG and placebo groups in response to hepatitis A ( $69 \%$ and $88 \%$, respectively, who tested negative before and positive after vaccination; $p=0.36$ ) or tetanus toxoid $(66 \%$ and $62 \%$, respectively, whose average anti-tetanus score increased $>$ twofold after revaccination). Thus, a single course of ATG therapy did not impair humoral immune responses to a neoantigen and memory responses to a recall antigen at 12 months when the circulating $\mathrm{T}$ cell compartment was partially reconstituted.

\section{Discussion}

Our study shows that a course of ATG administered within 100 days of type 1 diabetes diagnosis did not affect the rate of beta cell loss over 24 months, as compared with the placebo group. As noted in the 12-month report [10], this result was unexpected given the preclinical and clinical observations with ATG used alone or in combination with other agents [13-15].
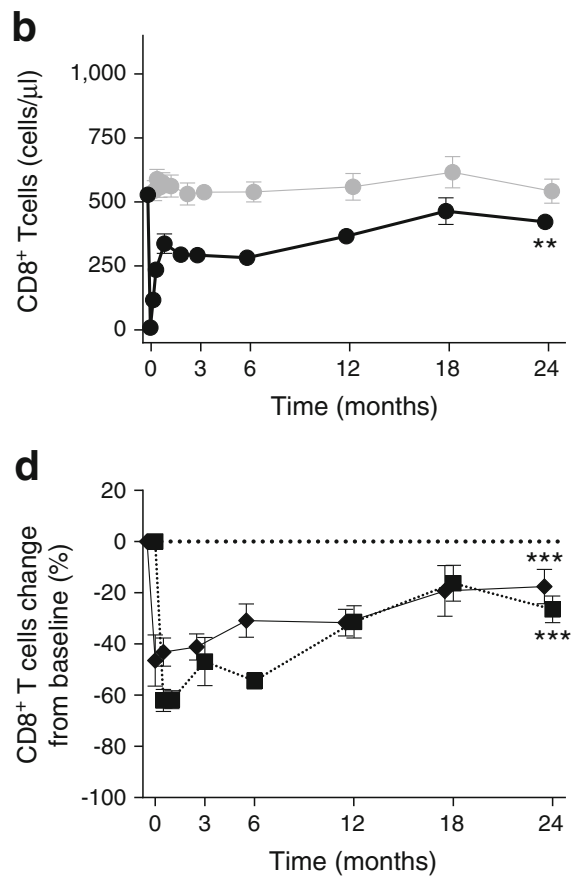

cells $\left(\mathrm{CD} 8^{+} \mathrm{CD} 45 \mathrm{RA}^{-} \mathrm{CD} 45 \mathrm{RO}^{+} \mathrm{CD} 62 \mathrm{~L}^{\text {hi }}\right)$. Black circles, ATG; grey circles, placebo in (a) and (b); diamonds, Tcm; inverted triangles, Treg; squares, Tn in (c) and (d). All data are mean values \pm SEM. Data were $\log _{2}$-transformed and analysed using one-sided paired $t$ tests to test whether the absolute cell counts at month 24 were significantly lower than baseline within the ATG group. ${ }^{* * *} p<0.001,{ }^{* *} p<0.01$

The kinetics of C-peptide decline observed over the first 12 months of this study were unusual in the ATG group, with a biphasic response: an initial decline in beta cell function, followed by stabilisation from months 6 to 12 . This early C-peptide decline may have resulted from unintended immune activation due to CRS and serum sickness [10]. We wondered if the stabilisation in C-peptide from month 6-12 would be sustained out to 24 months, but the results of this longer-term follow-up show that this was not the case in the ITT population. Interestingly, the overall response was driven in large part by the younger cohort (ages 12-21 years), because the older participants (22-35 years) showed relative stabilisation in stimulated C-peptide responses at 12 months [10], which continued and reached statistical significance at 24 months (Fig. 1a-c).

The apparent treatment benefit in the older age group prompted a post hoc responder analysis: we identified a group of nine ATG-treated participants with complete preservation of C-peptide at 2 years, of whom six (67\%) were in the older age group. This group of 'responders' clustered separately from the 'non-responders' in the ATG arm (Fig. 2a). However, there were also two placebo participants who met the threshold for response, suggesting that the responder phenotype may be a combination of slow disease progression and drug effect. Importantly, however, the responder group was predominantly composed of older participants, consistent with the analysis of C-peptide changes by age. 
In the ITT population, ATG treatment led to profound depletion of total lymphocytes and total $\mathrm{CD} 4^{+}$and $\mathrm{CD} 8^{+}$ $\mathrm{T}$ cells, with partial but incomplete recovery by 24 months. $\mathrm{CD}^{+}$and $\mathrm{CD} 8^{+} \mathrm{Tn}$ and Tcm cells and Tregs (but not Tem cells) were substantially depleted, again with partial but incomplete recovery by 24 months. Analysis by age group or by responder status, however, did not reveal any significant differences in depletion or reconstitution of any of these lymphocyte subsets.

It remains unclear why there was a dichotomy in C-peptide responses between the younger and older cohorts. This was a post hoc analysis in a small number of participants and therefore might be a chance finding. On the other hand, six of nine responders were in the older cohort, while there were no adult participants in the placebo 'responders', suggesting that age may indeed play a role. Consistent with the better preservation of C-peptide secretion in the older cohort, we also found significantly lower $\mathrm{HbA}_{1 \mathrm{c}}$ levels and lower exogenous insulin use in the older vs younger participants (ESM Fig. 2b, d). Type 1 diabetes is more aggressive in younger participants with a faster rate of C-peptide decline than in adults [16]. The underlying state of immune activation in type 1 diabetes may be age dependent, which may lead to differential outcomes after lymphodepletion with ATG. It is notable that ATG is used as an induction therapy in islet transplantation in adult recipients [17]. Interestingly, the 5-year insulin independence rates after islet transplantation are substantially higher in recipients who receive induction therapy with ATG plus a TNF- $\alpha$ inhibitor vs those who receive ATG alone, suggesting that it is important to block the ATG-induced CRS [17].

Despite substantial and prolonged T cell depletion (Fig. 3), there were no discernible safety concerns over the 2-year study period, aside from CRS and serum sickness around the time of the ATG infusion. Humoral responses to recall and neoantigens were preserved and there were no opportunistic infections in the ATG vs placebo groups. The comparatively good tolerability after the drug administration period may relate to the use of antimicrobial prophylaxis until CD4 counts were $>200$ cells $/ \mu \mathrm{l}$, incomplete T cell depletion with residual cells in the periphery, or the fact that other arms of the immune system remained intact.

Despite failure to achieve the primary endpoint, further development of ATG may be warranted. There is a potential benefit in older participants, which needs to be verified in larger trials. Of interest are the ongoing phase 1 clinical trials with autologous haematopoietic stem cell infusion using ATG in combination with granulocyte colony-stimulating factor (G-CSF) and cyclophosphamide [18-20]. This approach leads to better efficacy than any other immune intervention evaluated to date, but at the cost of significant short- and long-term morbidity [21]. A pilot study using $2.5 \mathrm{mg} / \mathrm{kg}$ ATG (one-third the dose used in the START trial) together with G-CSF for participants enrolled 4 months to 2 years from diagnosis showed a difference at 1 year between the treatment and placebo groups [22]. This success may have been due to: the lower dose of ATG, with less CRS and serum sickness; the use of G-CSF, with preservation of Tregs; or the inclusion of older participants, who may respond better to ATG. A phase 2 newonset trial evaluating lower-dose ATG with or without G-CSF is now under way (NCT02215200).

In conclusion, a single course of ATG infusions given during the new-onset period in type 1 diabetes had no significant effect on preservation of C-peptide secretion at 2 years compared with placebo in the ITT population. Treated participants experienced CRS and serum sickness during the peri-infusion period, but otherwise the drug was well tolerated. Post hoc analyses revealed significant preservation of C-peptide in older participants (age 22-35 years) and identified a subgroup of 'responders' predominantly comprising older patients. These results may warrant further studies with ATG in selected populations (e.g. older patients) or in combination with other immune modulators (e.g. G-CSF).

Acknowledgements We thank all the members of the ITN START Study Team, who are listed in the ESM.

Funding Research reported in this publication was supported by the National Institute of Allergy and Infectious Diseases (NIAID) of the National Institutes of Health (NIH) under award numbers NO1-AI15416 and UM1AI109565. The trial was conducted by the Immune Tolerance Network (ITN) and sponsored by the NIAID. Additional funding was provided by the National Institute of Diabetes and Digestive and Kidney Diseases (NIDDK), the JDRF, and multiple Clinical \& Translational Awards grants (NIH/NCRR UCSF-CTSI UL1TR000004, Minnesota-UL1TR000114, CHOP-NCRR UL1RR024134, CHOP-NCATS UL1TR000003, U. Colorado-CTSI UL1TR001082, UCSD N01-AI-15416, Emory-UL1TR000454 and CMH N01-AI-15416). Genzyme (Cambridge, MA, USA) provided ATG (Thymoglobulin ${ }^{\circledR}$ ) and LifeScan, Inc. (Milpitas, CA, USA) provided blood glucose monitoring meters and test strips.

Duality of interest statement SEG served as a consultant on an advisory board for Genzyme (2006). PAG reports grants from Pfizer, is a DSMB member for Viacyte, serves on the Scientific Advisory Board for Tolerion, received study drug from Glaxo Smith Kline, and received study drug from Bristol Meyers Squibb (all outside the submitted work); in addition, PAG has a patent 'Use of Methyldopa to treat T1D' licensed, a patent 'AAT to treat T1D' with royalties paid, a patent 'Small Molecules to treat T1D' licensed and a patent 'Preparation to improve Graft Survival' pending. EIF reports Drug Advisory Board activities for Novo Nordisk, Alexion and NPS (all outside the submitted work). All other authors declare that there is no duality of interest associated with their contribution to this manuscript.

Contribution statement SEG served as study chair. SEG, MRE, AP, LKE, KMH, DP, LD and JAB conceived and designed the study, including all statistical and analytical methodologies. SEG, PAG, EIF, SMW, LKF, AM, MG and WVM contributed to collection of the data. SEG, MRE, AP, LKE, KMH and SK analysed and interpreted the data. SEG, MRE, AP and KMH wrote the paper. All authors contributed to discussion, reviewed the manuscript critically for important intellectual content and approved the final version to be published. SEG is responsible for the integrity of the work as a whole. The content is solely the responsibility of the authors and does not necessarily represent the official views of the NIH. 


\section{References}

1. Bluestone JA, Herold K, Eisenbarth G (2010) Genetics, pathogenesis and clinical interventions in type 1 diabetes. Nature 464:12931300

2. The Canadian-European Randomized Control Trial Group (1988) Cyclosporin-induced remission of IDDM after early intervention: association of $1 \mathrm{yr}$ of cyclosporin treatment with enhanced insulin secretion. Diabetes 37:1574-1582

3. Feutren G, Papoz L, Assan R et al (1986) Cyclosporin increases the rate and length of remissions in insulin-dependent diabetes of recent onset. Results of a multicentre double-blind trial. Lancet 2:119-124

4. Orban T, Bundy B, Becker DJ et al (2014) Costimulation modulation with abatacept in patients with recent-onset type 1 diabetes: follow-up 1 year after cessation of treatment. Diabetes Care 37: 1069-1075

5. Keymeulen B, Vandemeulebroucke E, Ziegler AG et al (2005) Insulin needs after CD3-antibody therapy in new-onset type 1 diabetes. N Engl J Med 352:2598-2608

6. Sherry N, Hagopian W, Ludvigsson J et al (2011) Teplizumab for treatment of type 1 diabetes (Protege study): 1-year results from a randomised, placebo-controlled trial. Lancet 378:487-497

7. Herold KC, Gitelman SE, Ehlers MR et al (2013) Teplizumab (anti$\mathrm{CD} 3 \mathrm{mAb}$ ) treatment preserves $\mathrm{C}$-peptide responses in patients with new-onset type 1 diabetes in a randomized controlled trial: metabolic and immunologic features at baseline identify a subgroup of responders. Diabetes 62:3766-3774

8. Pescovitz MD, Greenbaum CJ, Krause-Steinrauf H et al (2009) Rituximab, B-lymphocyte depletion, and preservation of beta-cell function. N Engl J Med 361:2143-2152

9. Rigby MR, Harris KM, Pinckney A et al (2015) Alefacept provides sustained clinical and immunological effects in new-onset type 1 diabetes patients. J Clin Invest 125:3285-3296

10. Gitelman SE, Gottlieb PA, Rigby MR et al (2013) Antithymocyte globulin treatment for patients with recent-onset type 1 diabetes: 12-month results of a randomised, placebo-controlled, phase 2 trial. Lancet Diabetes Endocrinol 1:306-316

11. Amiel SA, Sherwin RS, Simonson DC, Lauritano AA, Tamborlane WV (1986) Impaired insulin action in puberty. A contributing factor to poor glycemic control in adolescents with diabetes. N Engl J Med 315:215-219
12. Miller KM, Foster NC, Beck RW et al (2015) Current state of type 1 diabetes treatment in the U.S.: updated data from the T1D Exchange clinic registry. Diabetes Care 38:971-978

13. Simon G, Parker M, Ramiya V et al (2008) Murine antithymocyte globulin therapy alters disease progression in NOD mice by a timedependent induction of immunoregulation. Diabetes 57:405-414

14. Eisenbarth GS, Srikanta S, Jackson R et al (1985) Anti-thymocyte globulin and prednisone immunotherapy of recent onset type 1 diabetes mellitus. Diabetes Res 2:271-276

15. Saudek F, Havrdova T, Boucek P, Karasova L, Novota P, Skibova J (2004) Polyclonal anti-T cell therapy for type 1 diabetes mellitus of recent onset. Rev Diabet Stud 1:80-88

16. Greenbaum CJ, Beam CA, Boulware D et al (2012) Fall in Cpeptide during first 2 years from diagnosis: evidence of at least two distinct phases from composite Type 1 Diabetes TrialNet data. Diabetes 61:2066-2073

17. Bellin MD, Barton FB, Heitman A et al (2012) Potent induction immunotherapy promotes long-term insulin independence after islet transplantation in type 1 diabetes. Am J Transplant 12:15761583

18. Couri CE, Oliveira MC, Stracieri AB et al (2009) C-peptide levels and insulin independence following autologous nonmyeloablative hematopoietic stem cell transplantation in newly diagnosed type 1 diabetes mellitus. JAMA 301:1573-1579

19. Li L, Shen S, Ouyang J et al (2012) Autologous hematopoietic stem cell transplantation modulates immunocompetent cells and improves beta-cell function in Chinese patients with new onset of type 1 diabetes. J Clin Endocrinol Metab 97:1729-1736

20. Snarski E, Milczarczyk A, Torosian T et al (2011) Independence of exogenous insulin following immunoablation and stem cell reconstitution in newly diagnosed diabetes type I. Bone Marrow Transplant 46:562-566

21. D'Addio F, Valderrama Vasquez A, Ben Nasr M et al (2014) Autologous nonmyeloablative hematopoietic stem cell transplantation in new-onset type 1 diabetes: a multicenter analysis. Diabetes 63:3041-3046

22. Haller MJ, Gitelman SE, Gottlieb PA et al (2015) Anti-thymocyte globulin/G-CSF treatment preserves beta cell function in patients with established type 1 diabetes. J Clin Invest 125:448-455 